\title{
Endoscopic Ultrasonography Miniature Probe Performance for Depth Diagnosis of Early Gastric Cancer with Suspected Submucosal Invasion
}

\author{
Hiroyuki Takamaru ${ }^{1}$, Shigetaka Yoshinaga ${ }^{1}$, Hajime Takisawa ${ }^{1}$, Ichiro Oda ${ }^{1}$, Hitoshi Katai ${ }^{2}$, Shigeki Sekine ${ }^{3}$, Kazuhiro \\ Taniguchi ${ }^{3}$, and Yutaka Saito ${ }^{1}$ \\ Divisions of ${ }^{1}$ Endoscopy, ${ }^{2}$ Gastric Surgery, and ${ }^{3}$ Pathology, National Cancer Center Hospital, Tokyo, Japan
}

\begin{abstract}
Background/Aims: The accurate assessment of the depth of invasion of early gastric cancer (EGC) is critical to determine the most appropriate treatment option. However, it is difficult to distinguish shallow submucosal (SM1) invasion from deeper submucosal (SM2) invasion. We investigated the diagnostic performance of endoscopic ultrasonography (EUS) using a miniature probe for EGC with suspected SM invasion. Methods: From April 2008 to June 2018, EGCs with suspected SM invasion were analyzed retrospectively. The EGCs examined by a $20 \mathrm{MHz}$ high-frequency miniature probe was included in our study. Esophago-gastric junction cancers and patients treated by chemotherapy before resection were excluded. The sensitivity and specificity for the detection of SM2 invasion by EUS were compared with those of white light imaging (WLI). Additionally, factors related to depth underestimation or overestimation were investigated using multivariate analysis. Results: A total of 278 EGCs in 259 patients were included in the final analysis. The sensitivity and specificity for SM2 or deeper by EUS were $73.7 \%(87 / 118)$ and $74.4 \%(119 / 160)$, respectively. The sensitivity and specificity by WLI were $47.5 \%$ (56/118) and 68.1\% (109/160), respectively. The sensitivity of EUS was significantly superior to that of conventional endoscopy $(p<0.01)$. Multivariate analysis revealed that an anterior location of the EGC was an independent risk factor for underestimation by EUS (odds ratio, 3.3; 95\% confidence interval, 1.1 to $9.8 ; p=0.03$ ). Conclusions: The depth diagnostic performance for EGCs with suspected SM invasion using EUS was satisfactory and superior to that of conventional endoscopy. Additionally, it is important to recognize factors that may lead to misdiagnosis in those lesions. (Gut Liver 2020;14:581-588)
\end{abstract}

\section{INTRODUCTION}

Accurate assessment of depth of invasion for early gastric cancer (EGC) is critical to select endoscopic resection (ER) or surgery as the appropriate treatment option.

EGCs confined to the mucosa (M) are candidates for ER, while those with the deep submucosal (SM2) invasion (more than 500 $\mu \mathrm{m})$ should undergo surgery. ${ }^{1}$ Additionally, considering the curative resection criteria for tumors of expanded indication, EGCs confined to the shallow layer (within $500 \mu \mathrm{m}$ from the muscularis mucosa) of the submucosa (SM1) can be resected by ER without the need for additional surgery. ${ }^{2}$ Therefore, the differential diagnosis between EGC of M or SM1 invasion and those with SM2 or deeper invasion is critical in determining the appropriate treatment strategy. Several studies have reported that endoscopic ultrasonography (EUS) using high-frequency ultrasound probes for depth invasion of EGC is more accurate than conventional EUS. ${ }^{3-5}$ Obvious M or SM2 EGCs can be diagnosed by conventional high-definition white light imaging (WLI) alone; however, in situations where it is difficult to distinguish between superficial EGC (M-SM1) or deeper EGC (SM2), additional EUS examination may be beneficial. Several previous studies have reported the accuracy of EUS to distinguish M and SM depth of invasion for lesions that are borderline M or SM compared to diagnosis for obvious intramucosal lesion (M) or obvious SM2 EGCs. ${ }^{6,7}$

In this study, we investigated the diagnostic performance of EUS using miniature probe for EGCs with suspected SM invasion.

Key Words: Endosonography; Stomach neoplasms; Invasion depth

Correspondence to: Hiroyuki Takamaru

Division of Endoscopy, National Cancer Center Hospital, 5-1-1 Tsukiji Chuo-ku, Tokyo 104-0045, Japan

Tel: +81-3-3542-3815, Fax: +81-3-3542-2511, E-mail: htakamar@ncc.go.jp

Received on July 17, 2019. Revised on September 10, 2019. Accepted on September 11, 2019. Published online December 11, 2019. pISSN 1976-2283 eISSN 2005-1212 https://doi.org/10.5009/gnl19243

(a) This is an Open Access article distributed under the terms of the Creative Commons Attribution Non-Commercial License (http://creativecommons.org/licenses/by-nc/4.0) which permits unrestricted non-commercial use, distribution, and reproduction in any medium, provided the original work is properly cited. 


\section{MATERIALS AND METHODS}

\section{Patients}

From April 2008 to June 2018, 1,209 consecutive patients with 1,999 EGCs suspicious of SM invasion diagnosed by endoscopic examination at our institution were analyzed retrospectively. This study was approved by National Cancer Center Hospital Ethics Committee (approval number: 2016-447). Among

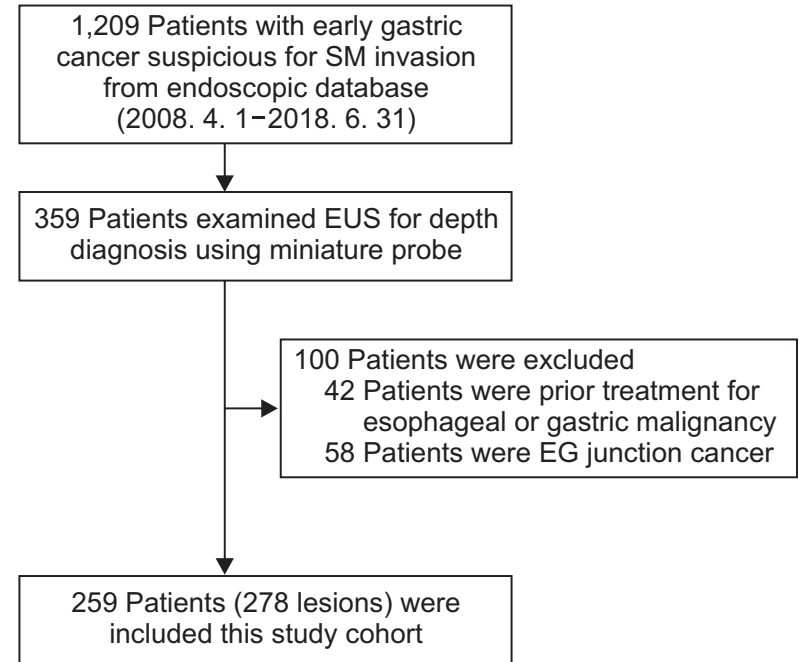

Fig. 1. Patient flowchart.

SM, submucosal; EUS, endoscopic ultrasonography; EG, esophagogastric. those, the EGCs examined by high-frequency miniature probe were included our study. The following cases were excluded: lesions located at the esophago-gastric junction (Siewert type 2) and lesions in patients who had received chemotherapy between examinations and resection (Fig. 1). The technique has been previously described in detail. Briefly, we performed EUS using $20 \mathrm{MHz}$ high-frequency ultrasound probes (UM-3R; Olympus Medical Systems, Tokyo, Japan). After washing the lesion, we filled the stomach with deaerated water through an endoscope channel. After the lesion was submerged, we performed ultrasound with the miniature probe. EUS was performed by three endoscopists (H. Takamaru, H. Takisawa, and S.Y.) on a separate day from the index WLI examination. All EUS findings were supervised by one expert endosonographer (S.Y.). The final depth diagnosis via EUS was agreed upon after discussion between the three endosonographers in this study. In addition, WLI examination for depth diagnosis and EUS examination for depth diagnosis was performed by a different endoscopist.

\section{Methods}

The endoscopic criteria for deep obvious SM2 EGCs by WLI were as follows: an uneven, irregular, or nodular surface, a marked margin elevation or submucosal tumor-like protrusion without flexibility, a deep ulceration, an irregular protrusion (in lesions with a 0-I component), a remarkable redness, and an enlarged, clubbing, or fused folds (Fig. 2). If the endoscopic findings listed above were identified, we decided to refer the patient
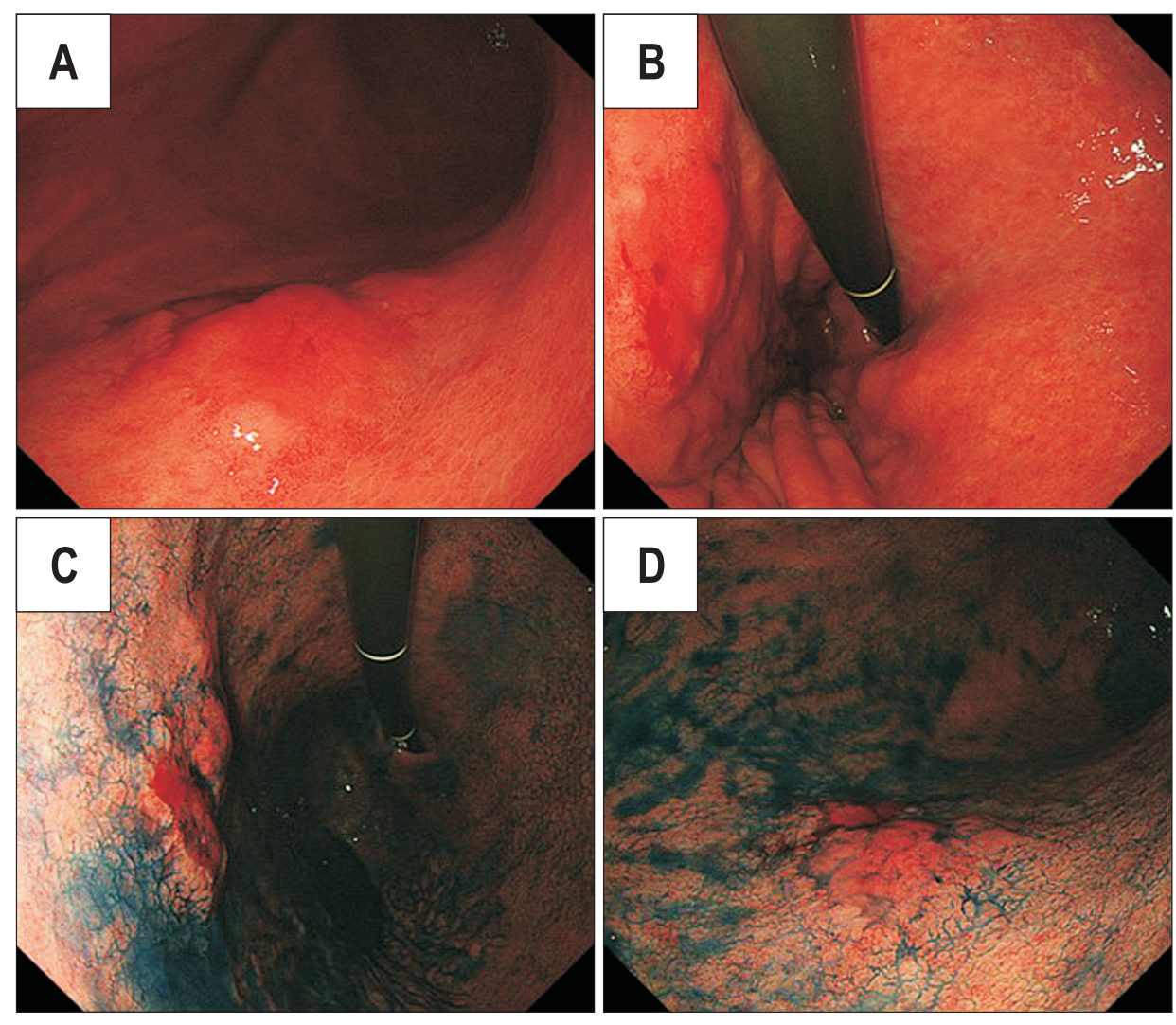

Fig. 2. Representative endoscopic findings of gastric cancer with deep submucosal invasion. The lesion showed an uneven, irregular, or nodular surface (A-D), an irregular protrusion $(\mathrm{A})$, a remarkable redness (A, B), and stiffness (B). 
for surgery. ${ }^{8,9}$ When the depth diagnosis based on the above endoscopic criteria was of lower confidence, (i.e., patients were judged as SM1 >SM2 or SM2>SM1) the endoscopists decided to perform EUS for a more detailed examination.

We reviewed endoscopic reports, electronic medical records, and histological results of EGCs. Endoscopic and histological findings evaluated were as follows: patient's age, sex, histologically defined size of the lesion, endoscopic morphology, direction of the wall upon which the lesion was located (anterior wall, posterior wall, lesser curvature, or greater curvature), location of the lesion in the stomach (upper-third, U; middle-third, M; or lower-third, L), histological differentiation, endoscopically or histologically diagnosed ulceration, estimated depth by conventional endoscopy or by EUS, and histological depth of invasion. The EUS criteria of M-SM1 EGCs was an intact 3rd layer (submucosal layer) or 3rd layer with irregularity. SM2 EGCs were defined as narrowing or an interruption of the 3rd layer (Fig. $3 \mathrm{~A}) .^{10-12}$ To differentiate submucosal fibrosis with submucosal invasion, we focused on the shape of the hypo-echoic image of the 2nd layer that narrowed the 3rd layer. If the hypo-echoic component showed a fan-like appearance we defined the narrowed 3rd layer to be due to submucosal fibrosis (Fig. 3B). The histological depth of invasion was considered the gold standard of depth diagnosis.

We calculated the sensitivity and specificity of WLI and EUS for SM2 or deeper for diagnostic performance to distinguish M-SM1 and SM2 or deeper. The sensitivity and specificity by EUS was compared to those by WLI. We investigated the factors related to the underestimation (i.e., histologically SM2 or deeper EGC but diagnosed M-SM1 by EUS) or overestimation (i.e., histologically M-SM1 EGC but diagnosed SM2 or deeper by EUS) using multivariate analysis. Co-variate factors were selected according to previous studies.

\section{Statistical analysis}

Results were expressed as mean \pm standard deviation (SD) or median and range. We used the McNemar test to compare the sensitivity and specificity of WLI and EUS diagnosis. The odds ratio and 95\% confidence interval were computed using binominal logistic regression analysis. A two-tailed p-value $<0.05$ was considered to be statistically significant. Statistical analysis was performed using the JMP SAS version 13.0.0 (SAS Institute, Cary, NC, USA).

\section{RESULTS}

A total of 278 EGCs in 259 patients were included for final analysis. Demographics and EGC data are listed in Table 1. The mean age of patients was 69.8 years old (SD, \pm 9.1 ). There were 197 males (76.1\%) and 62 females (23.9\%). Among all of the EGCs, the median size of tumors resected was $20.0 \mathrm{~mm}$ (range, 3.0 to $110.0 \mathrm{~mm}$ ). EGCs were located most commonly along the lesser curve 89 (32\%) and posterior wall of the stomach 75 (27\%). The prevalence of endoscopic and histological ulceration was 18.3\% and 16.9\%, respectively. About half of the EGCs (55.4\%) were treated endoscopically. More than half of the EGCs showed histology of differentiated type (66.2\%), followed by mixed type of differentiated predominant (21.2\%). Only a small number of EGCs with mixed type of undifferentiated predominant or undifferentiated type were also seen. Regarding the ratio of histological depth of invasion, M, SM1, SM2, and muscularis propria was $42.8 \%, 15.1 \%, 37.8 \%$, and 4.3\%, respectively.

Table 2 shows the diagnostic performance of WLI and EUS. The sensitivity of EUS was significantly superior to that of conventional endoscopy (73.7\% vs 47.5\%, p<0.01). In this study, diagnostic sensitivity, specificity and overall accuracy for SM2 or deeper by EUS was 73.7\% (87/118), 74.4\% (119/160), and 74.1\% (206/278), respectively. Alternatively, sensitivity, specificity, and overall accuracy by WLI was 47.5\% (56/118), 68.1\% (109/160), and 59.4\% (165/278), respectively.

Table 3 shows the ratio of EGCs misdiagnosed by EUS. Thirtyone EGCs were underestimated as SM1 invasion by EUS, while 41 EGCs overestimated as SM2 invasion. The mean size of EGCs showed no differences in each group. Cases of EUS underesti-
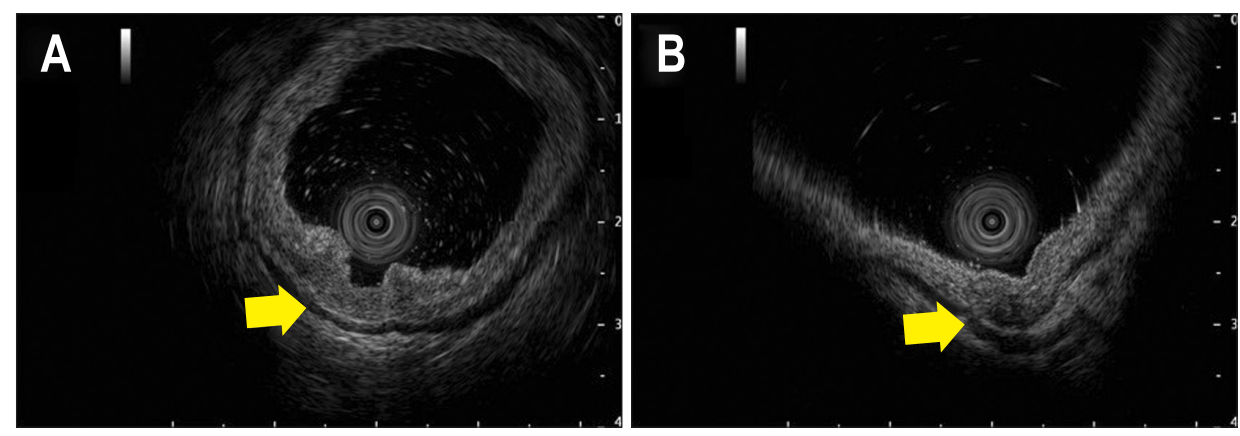

Fig. 3. Representative pictures of deep submucosal invasion and fibrosis. We diagnosed deep submucosal (SM2) invasion when endoscopic ultrasonography (EUS) showed narrowed or an interrupted 3rd layer (A; yellow arrow). On the other hand, we diagnosed submucosal fibrosis when a fan-like hypo echoic image (yellow arrow) was seen by EUS (B). 
Table 1. Demographic Data of the Patients and Lesions

\begin{tabular}{|c|c|}
\hline Variable & Value (\%) \\
\hline \multicolumn{2}{|l|}{ Sex } \\
\hline Male & 197 \\
\hline Female & 62 \\
\hline Age, mean $\pm S D, y r$ & $69.8 \pm 9.1$ \\
\hline Size of lesion size, median (IQR), mm & $20.0(3.0-110.0)$ \\
\hline \multicolumn{2}{|l|}{ Macroscopic feature } \\
\hline $0-\mathrm{I}$ & $23(8.2)$ \\
\hline 0-IIa & 99 (35.6) \\
\hline 0-IIb & $4(1.4)$ \\
\hline 0-IIc & $152(54.8)$ \\
\hline \multicolumn{2}{|l|}{ Location of the lesion } \\
\hline Upper & $103(37.1)$ \\
\hline Middle & $111(40.0)$ \\
\hline Lower & $64(23.0)$ \\
\hline \multicolumn{2}{|l|}{ Position in the gastric wall } \\
\hline Anterior wall & $46(16.5)$ \\
\hline Posterior wall & $75(27.0)$ \\
\hline Greater curvature & $68(24.5)$ \\
\hline Lesser curvature & 89 (32.0) \\
\hline \multicolumn{2}{|l|}{ Ulceration (endoscopic) } \\
\hline Positive & $51(18.3)$ \\
\hline Negative & $227(81.7)$ \\
\hline \multicolumn{2}{|l|}{ Ulceration (histological) } \\
\hline Positive & 47 (16.9) \\
\hline Negative & $231(83.1)$ \\
\hline \multicolumn{2}{|l|}{ Primary treatment } \\
\hline ESD & $154(55.4)$ \\
\hline Surgery & $124(44.6)$ \\
\hline \multicolumn{2}{|l|}{ Histology } \\
\hline Differentiate type & $184(66.2)$ \\
\hline Mixed/differentiated predominant & $59(21.2)$ \\
\hline Mixed/undifferentiated predominant & $17(6.1)$ \\
\hline Undifferentiated type & $18(6.5)$ \\
\hline \multicolumn{2}{|l|}{ Histological depth of invasion } \\
\hline Mucosal cancer (M) & $119(42.8)$ \\
\hline Shallow submucosal invasion (SM1) & $42(15.1)$ \\
\hline Deep submucosal invasion (SM2) & $105(37.8)$ \\
\hline Invasion to muscularis mucosa (MP) & $12(4.3)$ \\
\hline
\end{tabular}

We found 278 early gastric cancer lesions with 259 patients available for analysis.

IQR, interquartile range; ESD, endoscopic submucosal dissection.

mation tended to be located in the anterior wall of the stomach (22.6\% vs $14.6 \%$ ) and have 0-I type macroscopic feature (19.4\% vs 7.7\%), while a small number of lesions were located in lower-third of the stomach (9.7\% vs $24.7 \%$ ). In comparison, EGCs overestimated by EUS tended to be located also in the anterior wall of the stomach (33.3\% vs 15.2\%), 0-IIc type macroscopic feature (70.7\% vs 51.9\%) and were associated with the presence of endoscopic ulceration (29.3\% vs 14.8\%) (Fig. 4).

After adjustment by multivariate analysis, lesion location on the anterior wall was the independent risk factor for underestimation by EUS (odds ratio, 3.3; 95\% confidence interval, 1.1 to $9.8 ; p=0.03)$. We found no significant factors for overestimation by EUS (Table 4).

\section{DISCUSSION}

Our study results indicate that EUS has superior sensitivity for SM2 or deeper compared to WLI for EGC suspicious for SM invasion. Treatment options for EGC are determined by clinicopathological factors including the depth of invasion. Higher sensitivity for SM2 or deeper enable adequate decision making and thereby avoiding redundant ER.

The patients included in our study were all examined using $20 \mathrm{MHz}$ miniature probe during EUS examinations. This highfrequency miniature probe has higher resolution and less penetration compared to conventional EUS. This is most suitable for depth diagnosis for EGC. ${ }^{13-17}$

Multivariate analysis revealed that anterior lesion location was an independent risk factor of underestimation by EUS. We speculated that anterior lesion location was different from other locations in terms of EUS probe approach. The anterior wall might be a difficult location to approach the probe correctly, as the miniature probe should approach the EGC lesions horizontally (Fig. 5).

In several previous reports, the diagnostic performance of EUS has been evaluated by accuracy. ${ }^{6,14,16,18,19}$ However, accuracy depends on the prevalence of SM2 or deeper EGC in the study subjects. On the other hand, sensitivity and specificity could evaluate the diagnostic performance, irrespective of SM2 or deeper EGC prevalence. Therefore, we believe diagnostic performance by EUS was firmly evaluated in our study.

Several studies have mentioned the sensitivity for SM2 or deeper EGC by EUS ranging from $66.3 \%$ to $84.0 \%,{ }^{17,20,21}$ similar to the findings in our study.

Several previous reports have identified risk factors associated with diagnostic accuracy such as size of the lesion, protruded (0-I) type EGCs, location of the upper-third of the stomach (U), and ulceration for misdiagnosis by EUS..$^{15,16,19,20,22-25}$ One of the reasons why these findings were not significant in our study was partially due to the differences in our study subjects and EUS method. With regards to study subjects, we only included EGCs suspicious for SM invasion, while other studies have included a high number of muscularis propria gastric cancers. ${ }^{18,20,21,25}$ EUS examination is not necessary in practice for lesions in which the depth diagnosis is evident based on endoscopic evaluation because of time and procedure related costs. Our study analysis only focused on EGC suspicious of SM invasion. Therefore, we 
Table 2. Diagnostic Performance of WLI and EUS

\begin{tabular}{|c|c|c|c|c|c|}
\hline & \multicolumn{2}{|c|}{ WLI } & \multicolumn{2}{|c|}{ EUS } & \multirow{2}{*}{ p-value* } \\
\hline & M/SM1 & SM2/deeper & M/SM1 & SM2/deeper & \\
\hline \multicolumn{6}{|l|}{ Histology } \\
\hline M/SM1 (n=160) & $109(68.1)$ & $51(31.9)$ & $119(74.4)$ & $41(25.6)$ & 0.20 \\
\hline SM2/deeper (n=118) & $62(52.5)$ & $56(47.5)$ & $31(26.3)$ & 87 (73.7) & $<0.01$ \\
\hline Overall accuracy & & 59.4 & & 74.1 & \\
\hline Sensitivity for SM2 or deeper & & 47.5 & & 73.7 & \\
\hline Specificity for SM2 or deeper & & 68.1 & & 74.4 & \\
\hline PPV for SM2 or deeper & & 54.8 & & 79.3 & \\
\hline NPV for SM2 or deeper & & 63.7 & & 68.0 & \\
\hline
\end{tabular}

Data are presented as number (\%) or percent.

WLI, white light image; EUS, endoscopic ultrasound; M, mucosal cancer; SM1, shallow submucosal; SM2, deep submucosal; PPV, positive predictive value; NPV, negative predictive value.

*McNemar test.

Table 3. Rate of Endoscopic Findings Resulting in the Overestimation and Underestimation of Depth Diagnosis by Endoscopic Ultrasound

\begin{tabular}{|c|c|c|c|c|}
\hline Risk factor for under/overestimation & $\begin{array}{l}\text { Underestimation } \\
\qquad(\mathrm{n}=31)\end{array}$ & $\begin{array}{c}\text { Adequate or } \\
\text { overestimation }(n=247)\end{array}$ & $\begin{array}{l}\text { Overestimation } \\
\quad(\mathrm{n}=41)\end{array}$ & $\begin{array}{c}\text { Adequate or } \\
\text { underestimation }(\mathrm{n}=237)\end{array}$ \\
\hline Size of the lesion, $\mathrm{mm}$ & $21.3 \pm 12.5$ & $23.8 \pm 15.0$ & $22.2 \pm 13.0$ & $23.8 \pm 15.1$ \\
\hline \multicolumn{5}{|l|}{ Position in the gastric wall } \\
\hline Lesser curvature & $10(33.3)$ & $82(33.2)$ & $16(39.0)$ & 73 (30.8) \\
\hline Greater curvature & $7(22.6)$ & $61(24.7)$ & $6(14.6)$ & $62(26.2)$ \\
\hline Anterior wall & $7(22.6)$ & $36(14.6)$ & $10(33.3)$ & $36(15.2)$ \\
\hline Posterior wall & $7(22.6)$ & $68(27.5)$ & $9(22.0)$ & $66(27.9)$ \\
\hline \multicolumn{5}{|l|}{ Location of the lesions } \\
\hline Upper & $15(48.4)$ & $88(35.6)$ & $15(36.6)$ & $88(37.1)$ \\
\hline Middle & $13(41.9)$ & $98(39.7)$ & $18(43.9)$ & $93(39.2)$ \\
\hline Lower & $3(9.7)$ & $61(24.7)$ & $8(19.5)$ & $56(23.6)$ \\
\hline \multicolumn{5}{|l|}{ Macroscopic feature (predominant) } \\
\hline $0-\mathrm{I}$ & $6(19.4)$ & $17(7.7)$ & $1(2.4)$ & $22(9.3)$ \\
\hline 0-IIa & $9(29.3)$ & $90(36.4)$ & $11(26.8)$ & $88(37.1)$ \\
\hline 0-IIlb & $1(3.2)$ & $3(1.2)$ & 0 & $4(1.7)$ \\
\hline 0-IIc & $15(48.4)$ & $137(55.5)$ & $29(70.7)$ & $123(51.9)$ \\
\hline \multicolumn{5}{|l|}{ Ulceration status (endoscopic) } \\
\hline Ulcer (-) & $27(87.1)$ & 204 (82.6) & $29(70.7)$ & $202(85.2)$ \\
\hline Ulcer (+) & $4(12.9)$ & $43(17.4)$ & $12(29.3)$ & $35(14.8)$ \\
\hline
\end{tabular}

Data are presented as mean \pm SD or number $(\%)$.

analyzed only EGCs suspected to have SM invasion to simulate actual clinical practice. Because EGCs are generally smaller than advanced gastric cancer, there were no differences in lesion size in the underestimated, overestimated, and the adequately diagnosed groups (Table 3 ). In some previous reports, EUS examinations were performed by conventional EUS or miniature probe..$^{20,21,23}$ This is one of the reasons that the upperthird stomach location was not a risk factor of misdiagnosis in our study, because it is often difficult to scan lesions located in the upper-third of the stomach using conventional EUS, while we have used only the miniature probe for EUS examination. The "0-I" macroscopic subtype has also been reported to be a factor for misdiagnosis, ${ }^{13,15}$ however, macroscopic features had no relationship to misdiagnosis in our study. A few number of EGCs with 0-IIb were included our study cohort, which was rarely seen in other studies, which might also affect the results. Several previous studies have reported ulceration as one of the risk factors for misdiagnosis, ${ }^{4,13,14,19,20}$ while both endoscopic and histological ulceration did not reach statistical significance in our study. Because histological findings were not suitable for 

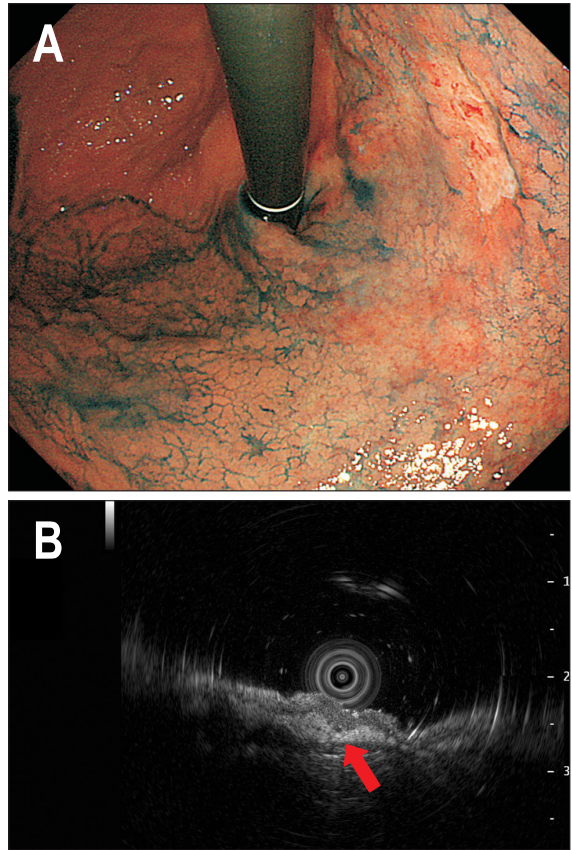

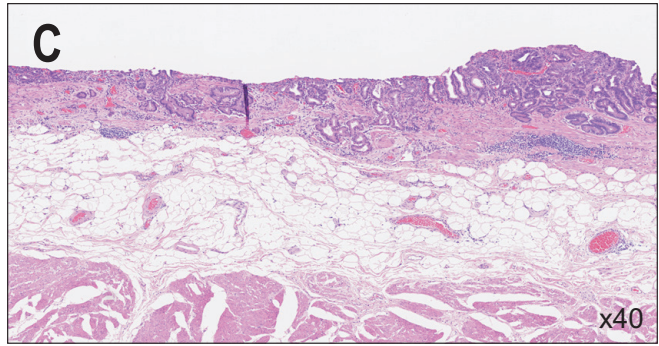

\section{D}

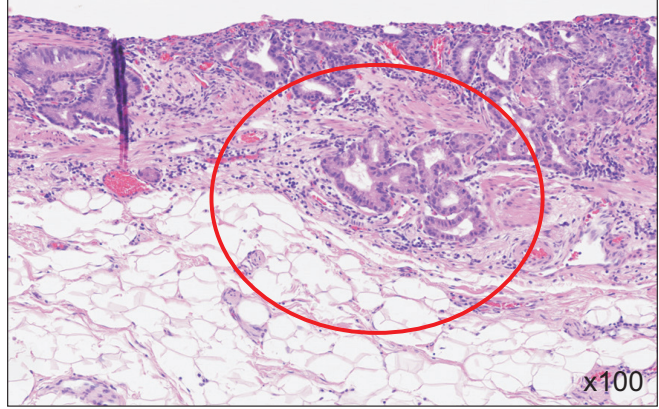

Fig. 4. Representative early gastric cancers (EGCs) that was overestimated by endoscopic ultrasonography (EUS) examination. (A) 0-IIC lesion was found in the upper-third, posterior wall of the stomach. (B) EUS revealed a narrowed 3rd layer (arrow) and diagnosed deep submucosal EGC. (C) Histological evaluation of surgically resected specimen revealed that the depth of invasion was pSM1, $370 \mu \mathrm{m}(\mathrm{H} \& \mathrm{E}, \times 40)$. (D) High power field of view (H\&E, $\times 100$ ). Histological diagnosis was as follows; tubular adenocarcinoma, well differentiated type, depth of invasion; pSM1 (red circle), $370 \mu \mathrm{m}$, ly0, v0, pPM 0, pDM 0.

pSM1, submucosal invasion, pathological; pPM, proximal margin, pathological; pDM, distal margin, pathological.

Table 4. Associated Endoscopic Findings of the Overestimation and Underestimation of Depth Diagnosis by Endoscopic Ultrasound

\begin{tabular}{|c|c|c|c|c|}
\hline \multirow{2}{*}{ Variable } & \multicolumn{2}{|c|}{ Underestimation } & \multicolumn{2}{|c|}{ Overestimation } \\
\hline & Multivariate OR (95\% CI) & p-value & Multivariate OR (95\% CI) & p-value \\
\hline \multicolumn{5}{|c|}{ Position in the gastric wall } \\
\hline Lesser curvature & 1 (reference) & & 1 (reference) & \\
\hline Greater curvature & $1.5(0.5-4.8)$ & 0.45 & $0.4(0.2-1.2)$ & 0.12 \\
\hline Anterior wall & $3.3(1.1-9.8)$ & $0.03^{*}$ & $1.3(0.5-3.3)$ & 0.56 \\
\hline Posterior wall & $1.1(0.4-3.4)$ & 0.87 & $0.7(0.3-1.6)$ & 0.37 \\
\hline \multicolumn{5}{|l|}{ Location of the lesions } \\
\hline Upper & 1 (reference) & & 1 (reference) & \\
\hline Middle & $0.8(0.3-1.9)$ & 0.60 & $0.9(0.4-2.0)$ & 0.83 \\
\hline Lower & $0.3(0.1-1.0)$ & 0.06 & $0.8(0.3-2.0)$ & 0.57 \\
\hline \multicolumn{5}{|c|}{ Macroscopic feature (predominant) } \\
\hline $0-\mathrm{I}$ & 1 (reference) & & 1 (reference) & \\
\hline 0-IIa & $0.4(0.1-1.2)$ & 0.11 & $3.0(0.4-25.3)$ & 0.31 \\
\hline $0-\mathrm{IIb}$ & $0.7(0.1-9.1)$ & 0.78 & $0.0(0.0-0.0)$ & 0.99 \\
\hline 0-IIc & $0.4(0.1-1.3)$ & 0.11 & $5.0(0.6-39.9)$ & 0.13 \\
\hline \multicolumn{5}{|c|}{ Ulceration status (endoscopic) } \\
\hline Ulcer (-) & 1 (reference) & & 1 (reference) & \\
\hline Ulcer (+) & $0.8(0.3-2.4)$ & 0.69 & $1.8(0.8-4.2)$ & 0.12 \\
\hline
\end{tabular}

$\mathrm{OR}$, odds ratio; $\mathrm{CI}$, confidence interval.

${ }^{*} \mathrm{p}<0.05$.

predictive factors prior to treatment, we used endoscopic ulceration in logistic regression analysis. To further assess the impact of ulceration, we also evaluated histological ulceration during post hoc analysis and found no correlation with misdiagnosis (Supplementary Table 1). One of the reasons for this discrepancy from previous reports is the number of lesions analyzed, as well as the differences of selected ECGs in our study.

We speculate that the 0-IIc macroscopic feature might work as a co-effective factor in logistic regression analysis, because both endoscopic and histological ulceration showed the highest ratio of 0-IIc (70.6\% and 74.5\% among all of lesions with ulceration, detail data not shown). 

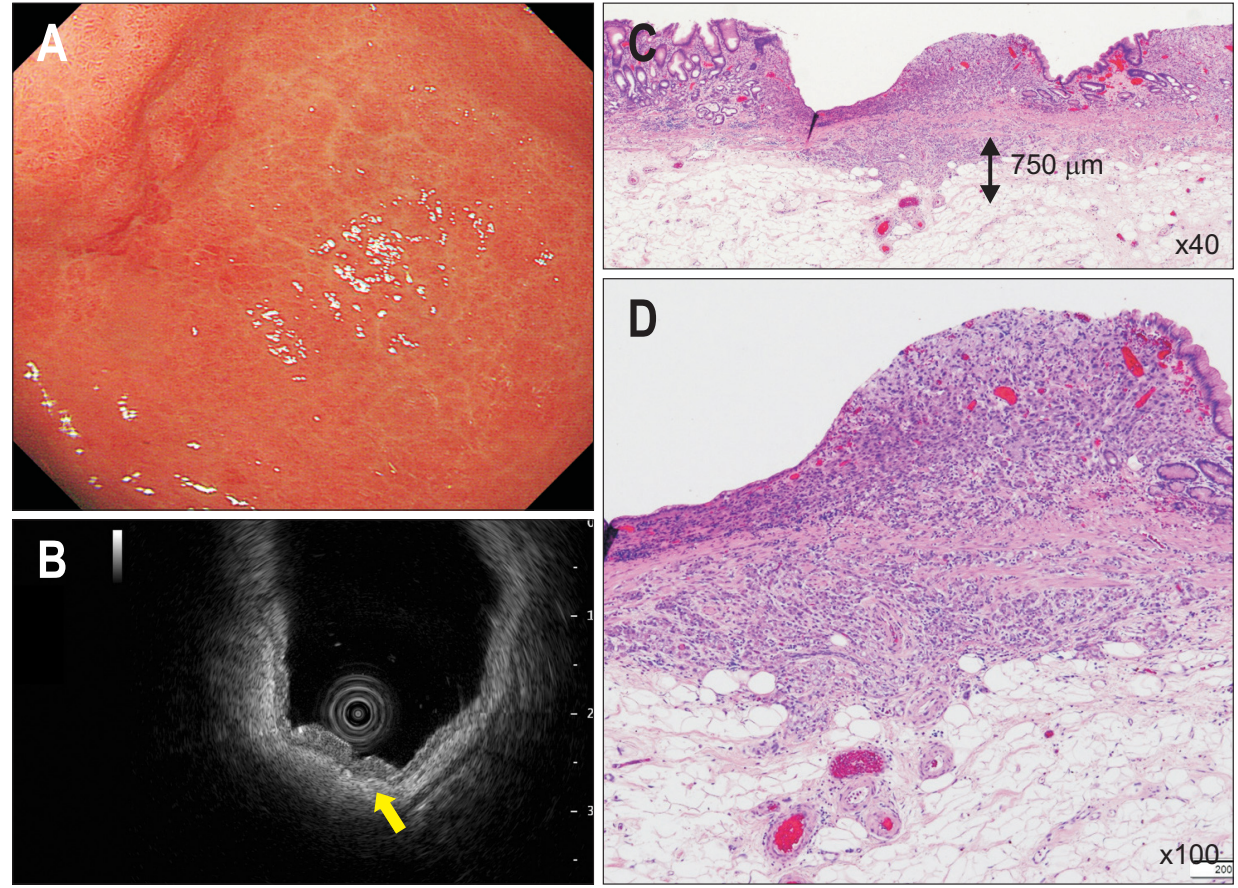

Fig. 5. Representative early gastric cancers (EGCs) located in the anterior wall of the stomach that was underestimated by endoscopic ultrasonography (EUS) examination. (A) 0-IIc lesion was found in the middle-third, anterior wall of the stomach. (B) EUS revealed the smooth 3rd layer (arrow) and diagnosed M-SM1 EGC. (C) Histological evaluation revealed that the depth of invasion was $\mathrm{pSM} 2,750 \mu \mathrm{m}(\mathrm{H} \& \mathrm{E}$, $\times 40$ ). (D) High power field of view $(\mathrm{H} \& \mathrm{E}, \times 100)$. Histological diagnosis was as follows; tubular adenocarcinoma, poorly differentiated type and signet ring cell carcinoma, depth of invasion: pSM2 $(750 \mu \mathrm{m})$, ly0, v0, pHM 0, pVM 0, ulceration (-).

M-SM1, intramucosal lesion-submucosal invasion; pSM2, submucosal invasion, pathological; pHM, horizontal margin, pathological; pVM, vertical margin, pathological.
On the viewpoint of practice, our study demonstrates the diagnostic performance of additional EUS examination followed by WLI diagnosis, because the WLI diagnosis was not blinded to each endosonographer. The additional EUS showed 28.2\% of overestimation of all overestimated patients while it was $42.3 \%$ by WLI alone (Supplementary Fig. 1). Similarly, EUS added after WLI showed only 5.7\% of underestimation while WLI underestimated 53.7\% (Supplementary Fig. 2). Taking into account these results, additional EUS examination adds value when the depth diagnosis is difficult by WLI only. Additionally, we have to be careful of overestimation even if it was diagnosed by EUS examination.

There are various limitations to the present study which warrant discussion. First, our study was a single center, retrospective study. Second, the diagnosis by WLI could not be blinded as practice, some kind of carry over effect is suspected and affects the result. On the other hand, in our institution, EUS diagnosis was supervised by one expert endosonographer. Therefore, uniform quality of diagnosis is ensured. ${ }^{17}$ Thirdly, only EGCs suspicious for SM invasion were analyzed, potentially leading to selection bias. The primary aim of EUS examination for EGCs is to determine the depth of tumor invasion and therefore determine the appropriate treatment option. Mocellin and Pasquali ${ }^{7}$ has reported that a study within a practical setting is important. In this point, our study was considered to be close to "real clinical practice." Another limitation to our study is histological evaluation after resection. In the current study, histological depth diagnosis was defined as the gold standard. For histological evaluation, sections were made in each $2 \mathrm{~mm}$ for ER and 5 $\mathrm{mm}$ in each for surgery. Different section widths could be con- sidered as potential bias.

In conclusion, this is the first report to identify the anterior wall as an independent risk factor to underestimate the depth diagnosis of EGC by EUS examination using high-frequency miniature probe. EUS examination with $20 \mathrm{MHz}$ miniature probe had higher sensitivity for EGC suspicious of SM invasion. As a result, EUS could reduce the incidence of redundant ER for SM2 invasive EGC with careful examination especially for EGCs located in anterior wall of the stomach.

\section{CONFLICTS OF INTEREST}

No potential conflict of interest relevant to this article was reported.

\section{ACKNOWLEDGEMENTS}

This work was supported in part by the National Cancer Center Research and Development Fund (25-A-12, 28-K-1, and 29A-13) to Y.S.

We would like to express our deepest gratitude to Dr. A. Aziz Aadam and Dr. Amar Manvar for writing support.

\section{AUTHOR CONTRIBUTIONS}

Study concept and design: H.Takamaru, S.Y. Data acquisition: H.Takamaru., S.Y., H.Takisawa, I.O., H.K., S.S., K.T., Y.S. Data analysis and interpretation: H.Takamaru., S.Y., S.S., K.T. Drafting of the manuscript: H.Takamaru., S.Y. Critical revision of the manuscript for important intellectual content: S.Y., K.T., Y.S. Statistical analysis: 
H.Takamaru. Obtained funding: Y.S. Study supervision: S.Y., I.O., Y.S.

\section{ORCID}

Hiroyuki Takamaru

Shigetaka Yoshinaga

Hajime Takisawa

Ichiro Oda

Hitoshi Katai

Shigeki Sekine

Kazuhiro Taniguchi

Yutaka Saito

\section{REFERENCES}

1. Japanese Gastric Cancer Association. Japanese gastric cancer treatment guidelines 2014 (ver. 4). Gastric Cancer 2017;20:1-19.

2. Hasuike N, Ono H, Boku N, et al. A non-randomized confirmatory trial of an expanded indication for endoscopic submucosal dissection for intestinal-type gastric cancer (cT1a): the Japan Clinical Oncology Group study (JCOG0607). Gastric Cancer 2018;21:114-123.

3. Cheng $\mathrm{J}$, Wu X, Yang A, et al. Model to identify early-stage gastric cancers with deep invasion of submucosa based on endoscopy and endoscopic ultrasonography findings. Surg Endosc 2018;32:855-863.

4. Park JS, Kim H, Bang B, Kwon K, Shin Y. Accuracy of endoscopic ultrasonography for diagnosing ulcerative early gastric cancers. Medicine (Baltimore) 2016;95:e3955.

5. Pei Q, Wang L, Pan J, Ling T, Lv Y, Zou X. Endoscopic ultrasonography for staging depth of invasion in early gastric cancer: a meta-analysis. J Gastroenterol Hepatol 2015;30:1566-1573.

6. Mouri R, Yoshida S, Tanaka S, Oka S, Yoshihara M, Chayama $\mathrm{K}$. Usefulness of endoscopic ultrasonography in determining the depth of invasion and indication for endoscopic treatment of early gastric cancer. J Clin Gastroenterol 2009;43:318-322.

7. Mocellin S, Pasquali S. Diagnostic accuracy of endoscopic ultrasonography (EUS) for the preoperative locoregional staging of primary gastric cancer. Cochrane Database Syst Rev 2015;(2):CD009944.

8. Abe S, Oda I, Shimazu T, et al. Depth-predicting score for differentiated early gastric cancer. Gastric Cancer 2011;14:35-40.

9. Choi J, Kim SG, Im JP, Kim JS, Jung HC, Song IS. Endoscopic prediction of tumor invasion depth in early gastric cancer. Gastrointest Endosc 2011;73:917-927.

10. Kim GH, Park DY, Kida M, et al. Accuracy of high-frequency catheter-based endoscopic ultrasonography according to the indications for endoscopic treatment of early gastric cancer. J Gastroenterol Hepatol 2010;25:506-511.

11. Kida M, Tanabe S, Watanabe M, et al. Staging of gastric cancer with endoscopic ultrasonography and endoscopic mucosal resec- tion. Endoscopy 1998;30 Suppl 1:A64-A68.

12. Yanai H, Matsumoto Y, Harada T, et al. Endoscopic ultrasonography and endoscopy for staging depth of invasion in early gastric cancer: a pilot study. Gastrointest Endosc 1997;46:212-216.

13. Yoshinaga S, Oda I, Nonaka S, Kushima R, Saito Y. Endoscopic ultrasound using ultrasound probes for the diagnosis of early esophageal and gastric cancers. World J Gastrointest Endosc 2012;4:218-226.

14. Kim SJ, Choi CW, Kang DH, et al. Factors associated with the efficacy of miniprobe endoscopic ultrasonography after conventional endoscopy for the prediction of invasion depth of early gastric cancer. Scand J Gastroenterol 2017;52:864-869.

15. Watari J, Ueyama S, Tomita $\mathrm{T}$, et al. What types of early gastric cancer are indicated for endoscopic ultrasonography staging of invasion depth? World J Gastrointest Endosc 2016;8:558-567.

16. Razavi SM, Khodadost M, Sohrabi M, et al. Accuracy of endoscopic ultrasonography for determination of tumor invasion depth in gastric cancer. Asian Pac J Cancer Prev 2015;16:3141-3145.

17. Kim TY, Yi NH, Hwang JW, Kim JH, Kim GH, Kang MS. Morphologic pattern analysis of submucosal deformities identified by endoscopic ultrasonography for predicting the depth of invasion in early gastric cancer. Surg Endosc 2019;33:2169-2180.

18. Lee HH, Lim CH, Park JM, et al. Low accuracy of endoscopic ultrasonography for detailed T staging in gastric cancer. World J Surg Oncol 2012;10:190.

19. Okada K, Fujisaki J, Kasuga A, et al. Endoscopic ultrasonography is valuable for identifying early gastric cancers meeting expandedindication criteria for endoscopic submucosal dissection. Surg Endosc 2011;25:841-848.

20. Kim J, Kim SG, Chung H, et al. Clinical efficacy of endoscopic ultrasonography for decision of treatment strategy of gastric cancer. Surg Endosc 2018;32:3789-3797.

21. Ikoma N, Lee JH, Bhutani MS, et al. Preoperative accuracy of gastric cancer staging in patient selection for preoperative therapy: race may affect accuracy of endoscopic ultrasonography. J Gastrointest Oncol 2017;8:1009-1017.

22. Yamamoto S, Nishida T, Kato M, et al. Evaluation of endoscopic ultrasound image quality is necessary in endosonographic assessment of early gastric cancer invasion depth. Gastroenterol Res Pract 2012;2012:194530.

23. Mandai K, Yasuda K. Accuracy of endoscopic ultrasonography for determining the treatment method for early gastric cancer. Gastroenterol Res Pract 2012;2012:245390.

24. Tsuzuki T, Okada H, Kawahara Y, et al. Usefulness and problems of endoscopic ultrasonography in prediction of the depth of tumor invasion in early gastric cancer. Acta Med Okayama 2011;65:105-112.

25. Park JM, Ahn CW, Yi X, et al. Efficacy of endoscopic ultrasonography for prediction of tumor depth in gastric cancer. J Gastric Cancer 2011;11:109-115. 